\title{
ELECTRON IMPACT EXCITATION OF Fe II AND BORON-LIKE IONS
}

\section{A. K. PRADHAN}

Department of Astronomy, Ohio State University, Columbus, ohio 43210 , U.S.A.
ABSTRACT: The present report consists of two separate items on
A. Collisional data for Fe II and,
B. Collisional data for Boron-like ions.

\section{A. Excitation of Fe II}

During the past decade a few pioneering calculations have been carried out for the extremely complex case of $\mathrm{Fe}$ II. The first set of calculations was done using the distorted wave approximation by Nussbaumer and Storey /1/ and Nussbaumer et al $/ 2 /$ including the lowest four states $3 d^{6}\left({ }^{5} D\right) 4 s\left[a^{6} D, a^{4} D\right]$, and $3 d^{7}\left[a^{4} F, a^{4} P\right]$ and the sextet odd parity states $z^{6} D^{\circ}, z^{6} F^{\circ}$ and ${ }^{6} P^{\circ}$. Fine structure was considered employing an algebraic transformation from LS coupling to pair coupling. Owing to the size of the calculations only one to three energies were considered and resonances were neglected.

The next major step in the Fe II computations was made by the Belfast group using the R-matrix method ( Baluja et al. $/ 3 /$, Berrington et al. /4/). However they were still unable to include more than a few states in the close coupling expansion as the calculations, with the required configuration expansion, were very large even on the Cray-XMP. Resonances were explicitly delineated in these calculations and some problems were noticed by Berrington et al. in the earlier work by Baluja et al.

An extension of the non-relativistic R-matrix package was made by Scott and Taylor /5/ to incorporate relativistic effects in the Breit-Pauli (BP) approximation to solve the scattering problem. Berrington et al. employed the BP R-matrix package for the fine structure collisition strengths.

Recently Berrington and Pradhan /6/ have carried out two sets of R-matrix calculations on the Cray- 2 which has a very large memory; approximately $90 \mathrm{MW}$ were needed for all of the calculations. The two sets consisted of (i) a 38-state LS coupling calcualtion and (ii) a 41-state fine structure BP calculation. In (i) a large number of states are included in the eigenfunction expansion dominated by the configurations $3 d^{6} 4 s, 3 d^{7}$ and $3 d^{6} 4 p$. Transitions among these states result in a number of prominent Infra-red and UV lines in the Fe II spectra. The Berrington and Pradhan calculations are at several hundred energies and delineate the resonance structures in the collision strengths for all transitions in both sets of calculations. The second set of BP calculations included the 41 fine structure sub-states in the lowest four states $3 d^{6} 4 s\left[{ }^{6} D,{ }^{4} D\right], 3 d^{7}\left[{ }^{4} F{ }^{4} P\right]$ and odd parity sextet and quartet states $3 d^{6}\left({ }^{5} D\right) 4 P\left[{ }^{6} D^{\circ},{ }^{6} F^{\circ},{ }^{6} P^{\circ},{ }^{4} F^{\circ o},{ }^{4} D^{\circ},{ }^{4} P^{\circ}\right]$.

Fig. 1 from Berrington and Pradhan shows the collision strength for the $a^{6} D-a^{4} F$ transition form the 38-state LS calculations. The heavy dots are the earlier results of Nussbaumer and Storey /4/. As can be seen the extensive resonance structures, particularly in the near threshold region, would likely affect the excitation rate siginificantly and the rate coefficient is expected to be much higher that given by the Nussbaumer and Storey values. Fig. 2 presents the collision strength for the 

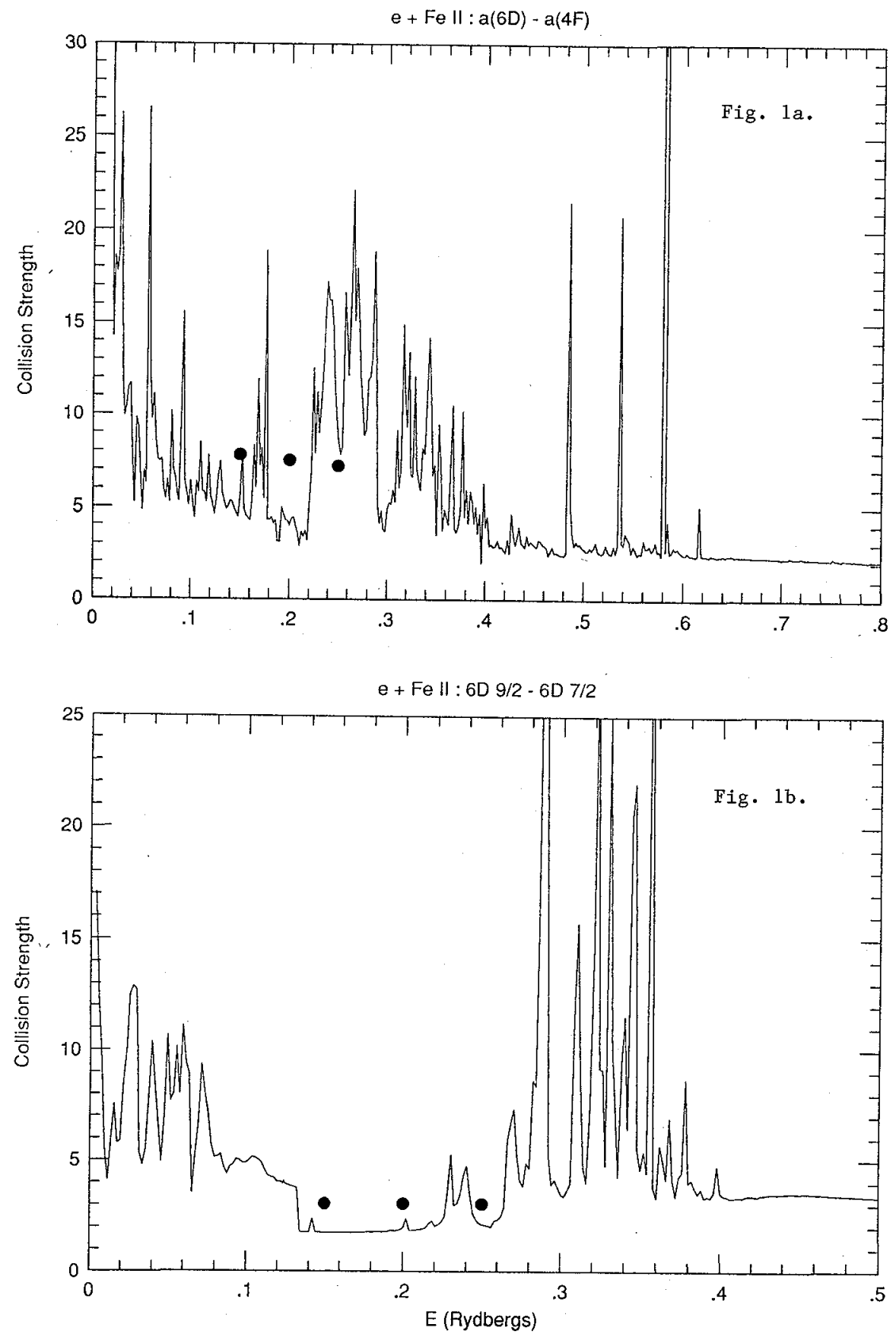
transition ${ }^{6} D_{9 / 2}-{ }^{6} D_{7 / 2}$ from the 41 -state fine structure calculation. Again, the near threshold resonance structure results in a much larger effective collision strength than the Nussbaumer et al. /6/ results.

A third set of calculations is currently in progress involving a pair coupling transformation of the reactance matrices from the 38-state LS calculation using a new code ,STGFJ, that is interfaced with the R-matrix package and is analogous to the program JAJOM by Saraph $/ 7 /$. It is expected that this calculation would yield collisional data for approximately 10,000 transition in Fe II. This work and the work on a detailed comparison of relativistic and non-relativistic results is in progress.

Based on preliminary comparisons, the earlier Nussbaumer and Storey and Nussbaumer et al. collision strengths appear to be considerably lower, due mainly to the neglect of resonance effects. For the forbidden transitions where such effects are more significant, their values result in rate coefficients that are upto a factor of 2 or 3 lower than the Berrington and Pradhan values. However, for the allowed transitions the two calculations appear to differ by less than $30 \%$.

\section{B. Excitation of Boron-like ions}

A comparison has been made of the collision data by the following authors : Hayes 18/, Hayes and Nussbaumer /9/, Lennon et al. /10/ and Luo and Pradhan /11/. All four calculations are in the close coupling approximation but differ in the number of states included. The first two calculations by Hayes consider O IV and C II respectiviely in a 5-state approximation using the IMPACT packacge of codes from University College London. Lennon et al. have considered C II in an 8-state calculation using the R-matrix method. The Hayes and Nussbaumer calculation for $\mathrm{C}$ II also considers the fine structure treansitions using the pair coupling transformation scheme by Saraph $/ 7 /$.

The most recent calculation using the R-matrix method, by Luo and Pradhan considers the first three ions in the Boron sequence : C II, N III and O IV. The 10 lowest states of C II and the 8 lowest states of N III and O IV are included in the target exapnsion. Including fine structure the collision strengths are calculated for all transitions among the states $2 s^{2} 2 p\left({ }^{2} P_{1 / 2,3 / 2}^{o}\right), 2 s 2 p^{2}$ $\left({ }^{4} P_{1 / 2,3 / 2,5 / 2},{ }^{2} D_{3 / 2,5 / 2},{ }^{2} S_{1 / 2},{ }^{2} P_{1 / 2,3 / 2}\right), 2 p^{3}\left({ }^{4} S_{3 / 2}^{o},{ }^{2} D_{3 / 2,5 / 2}^{o},{ }^{2} P_{1 / 2,3 / 2}^{o}\right), 2 s^{2} 3 s\left({ }^{2} S_{1 / 2}\right)$ and $2 s^{2} 3 p\left({ }^{2} P_{1 / 2,3 / 2}^{o}\right)$ for $\mathrm{C}$ II and among all states of $\mathrm{N}$ III and O IV excluding the $\mathrm{n}=3$ states. The fine structure collision strengths are calculated using the program STGFJ.

A comparison of the four different sets of calculations discussed above shows good agreement between collision strengths and rate coefficients, generally to within $20 \%$.

Acknowledgements : This work was supported in part by a grant from the U.S. National Science Foundation (Astronomy) grant no. AST-8996215. The computational work was carried out at the U.S. National Centre For Supercomputer Applications in Urabna, Illinois and at the Ohio Supercomputer Centre in Columbus, Ohio.

\section{REFERENCES :}

1. Nussbaumer, H. and Storey, P.J.,Astron.Astrophys.89,308-313(1980).

2. Nussbaumer, H.,Pettini,M. and

Storey,P.J.,Astron.Astrophys.102,351-358(1981). 
3. Baluja,K.L., Hibbert,A. and Mohan,M., J.Phys.B:At.Mol.Phys. 19,3613-23(1986).

4. Berrington,K.A., Burke,P.G., Hibbert,A., Mohan,M. and Baluja,K.L., J.Phys.B:At.Mol.Phys. 21,339-350(1988).

5. Scott,N.S. and Taylor,K.T., Comput.Phys.Commun. 25,347-387(1982).

6. Berrington,K.A. and Pradhan,A.K., J.Phys.B:At.Mol.Phys. (to be submitted).

7. Saraph,H.E., Comput.Phys.Commun. 15,247(1978); 3,256(1972).

8. Hayes,M. J.Phys.B:At.Mol.Phys. 16,285(1983).

9. Hayes,M. and Nussbaumer,H. Astron.Astrophys. 134,193(1984).

10. Lennon,D.J., Dufton,P.L., Hibbert,A. and Kingston,A.K., Astrophys.J. 294,200(1985).

11. Luo,D., and Pradhan,A.K., Phys.Rev.A 41,41(1990). 